\title{
Die verbotene „Handlung“ des Zinngießers Taberger oder der kleine Sarg des Anstoßes - Ein Fall außersprachlicher Kommunikation im Königreich Westphalen
}

Ende Februar 1813 wurde General Bongars, dem damaligen Chef der Gendarmerie und der politischen Polizei, ein Vorfall aus Hannover bekannt. Am 28. Februar ordnete er daraufhin eine nähere Untersuchung an: „un nommé Taberger, Polier d'étain a exposé aux regards du Public des militaires français et au milieu d'eux un petit cercueil en etain portant pour marque extérieure les Couleurs Tricolores de france. ... il y a ici une intention non equivoque qui mérite une punition exemplaire “. ${ }^{1} \mathrm{Die}$ Abschrift der bereits zuvor eingeleiteten Ermittlungen gegen Taberger durch die hannoverschen Polizeikommissare Grahn und Frömbling erhielt der Polizeichef am 4. März, und diese bildet die Grundlage für die Rekonstruktion der Affäre Taberger, die in die nonverbalen Kommunikationsformen der Westphalen einführen soll. ${ }^{2}$ Eine Leitfrage wird im folgenden sein, ob eine Sprachbarriere bestand und ob diese die Kommunikation zwischen den Französischsprachigen und Deutschsprachigen im Königreich Westphalen entscheidend behinderte.

Über die Identität Tabergers enthalten die Dokumente nur wenige sporadische Angaben. Er betrieb in Hannover an der sogenannten Langen Straße ein Familienhandwerk mit Tradition. Nach seinen eigenen Angaben war bereits sein Großvater Zinngießer. Der Bürger Taberger, der „bimbelotteries en étain de tout genre“ verkaufte, war ledig, kam als Hausbesitzer seiner Einquartierungspflicht nach, indem er französischen Soldaten Unterkunft gewährte, und fiel, so der Polizeikommissar Frömbling, bis zu dem hier zu behandelnden Vorfall mit seinem Benehmen nicht weiter auf. ${ }^{3}$

1 Schreiben von Bongars an Frantz, den Präfekten des Aller-Departements, 28. Febr. 1813, RNB, F 993 Arch. Westf., $\mathrm{N}^{\circ} 13850$, Registre $\mathrm{N}^{\circ} 1$ de correspondance du Bureau de la police secrète, commencé le 2 janvier et fini le 18 avril 1813.

2 Vgl. Bericht der Polizeikommissare Frömbling und Grahn an Bongars, 4. März 1813, HStAH, Hann. 52, Nr. 812, Bl. 180; Abschrift der Polizeiprotokolle betr. den Zinngießer Taberger, 22. Febr. und 2. März 1813, ebd., Bl. 181-186. - Die Untersuchung ordnet sich in das Thema meiner Dissertation „'der französischen Sprache mächtig ...'. Kommunikation im Spannungsfeld von Sprachen und Kulturen im Königreich Westphalen (1807-1813)“ ein, die innerhalb des Graduiertenkollegs „Interkulturelle Kommunikation in kulturwissenschaftlicher Perspektive“ der Universität des Saarlandes unter Betreuung in cotutelle von Prof. Dr. Rainer Hudemann (Saarbrücken) und Prof. Dr. Etienne François (Paris/Berlin) entstand und im Juli 2007 eingereicht wurde. Die Arbeit berücksichtigt insbesondere die Felder der Sprachpolitik, der Sprachpraxis und des Sprachbewußtseins in der Gesellschaft des Königreichs Westphalen. Zum Forschungshorizont im Feld der Sozial- und Kulturgeschichte der Sprache, des Sprechens und der Kommunikation vgl. Burke, Küchenlatein, S. 8, 10; Schlieben-Lange, Traditionen; ferner Spillner, Sprache, S. 8 (Vorwort). Von meinen Forschungen bereits veröffentlicht: Paye, Sprach- und Zensurpolitik.

3 Bericht der Polizeikommissare Grahn und Frömbling an Bongars, 23. Febr. 1813, RNB, F 993 Arch. Westf., K. 13, $\mathrm{N}^{\circ} 7604-7687$, hier $\mathrm{N}^{\circ} 7637$; vgl. auch Abschrift der Polizeiprotokolle betr. den Zinngießer Taberger, 22. Febr. und 2. März 1813, HStAH, Hann. 52, Nr. 812, B1. 181-186. 
Am 22. Februar erhielt jedoch die hannoversche Polizei einen Hinweis und vergewisserte sich selbst des Vergehens Tabergers: „Auf erhaltene Anzeige, daß ... Taberger, ... unter denen hinter seinen Fenstern aufgestellten in Zinn oder Blei abgegossenen Soldaten und Officieren der französischen Armee - namentlich von einem Trupp französischer Officiere zu Pferde, worunter Mameluken wären - auf eine anstößige Art einen kleinen Sarg aufgestellt habe, verfügte ich ... mich selbst persönlich nach dem Hause des Herrn Taberger". ${ }^{4}$ Die Beobachtungen des Polizeikommissars Frömbling vor Ort waren noch genauer als die präzisen Angaben des Denunzianten. „Ich erblickte ... hinter dem Fenster mehreres französisches Militair, in Blei abgegossen, aufgestellt. Unter diesen standen auf einem Fleck unsere französischen Officiere zu Pferde nebst 2 Mameluken, welches die hiesige Escadron vorstellen konnte, und ganz nahe vor diesen letzteren und eben so nahe hinter andern, - also zwischen diesen Militairs, stand ein kleiner schwarzer Sarg. ... Bei näherer Ansicht des Sarges bemerkte ich, daß auch die französische National-Farbe an demselben angebracht war" ${ }^{5}$ Mit der Identifizierung der französischen Nationalfarben am kleinen Sarg erschien dem Polizeikommissar das Delikt offensichtlich, und es folgte unmittelbar die Konfrontation Tabergers mit seiner Tat. Die Verhörprotokolle bilden die Basis zur Ermittlung der Motivation und Rechtfertigung des überführten Zinngießers und ermöglichen, auf die Interpretation seiner Tat durch die Polizei näher einzugehen. ${ }^{6}$

\section{Interpretation der Sarginszenierung im Schaufenster des Zinngießers Taberger}

Tatort war ein Schaufenster an der Langen Straße in der 17.000 Einwohner zählenden Stadt Hannover. ${ }^{7}$ Die Aufstellung französischer Bleisoldaten und eines Sarges hatte also unbestreitbar einen Ausstellungs- und Öffentlichkeitscharakter. Die Tatsache, daß Taberger den von Soldaten umstellten Sarg ausgerechnet „öffentlich vor seinem Fenster zur Schau ausgestellt habe", machte seinen Fall in den Augen der hannoverschen Polizeikommissare besonders brisant und zu einer Angelegenheit der politischen Polizei. ${ }^{8}$

Frömbling betonte in seinem Polizeibericht vom 22. Februar, daß ihm „die Aufstellung dieses Sarges unter den Umständen höchst anstößig“ vorkam. ${ }^{9}$ Die „Umstände“ im Februar 1813 waren folgende: in Hannover trafen seit der zweiten Februarwoche Tag für Tag Überlebende des Rußlandfeldzugs ein, wie aus einem anderen Polizeibericht Frömblings und Grahns über die solidarischen Bekundungen der Hannoveraner gegenüber diesen Soldaten hervorgeht. Am 16. Februar meldeten sie, daß die für zweihundert Kranke vorgesehenen militärischen Krankenhäuser der Stadt allein

4 Abschrift des Polizeiprotokolls betr. den Zinngießer Taberger, 22. Febr. 1813, HStAH, Hann. 52, Nr. 812, B1. $185 f$.

5 Ebd.

6 Ebd., B1. 181f.

7 Vgl. Hof- und Staats-Handbuch, S. 150.

8 Abschrift des Polizeiprotokolls betr. den Zinngießer Taberger, 22. Febr. 1813, HStAH, Hann. 52, Nr. 812, B1. 181f.

9 Ebd., B1. $185 f$. 
schon über achthundert Soldaten aufnehmen mußten, so daß um die hundert weitere aus Platzmangel bei Bürgern der Stadt untergebracht werden mußten. Die Hannoveraner aller Gesellschaftsschichten würden ungeachtet der Last und der Seuchengefahr ihre Hilfe durch materielle Unterstützung und Zuwendung freiwillig anbieten. ${ }^{10}$

Um jedoch trotz dieser weit verbreiteten Wohltätigkeit und Barmherzigkeit der Hannoveraner Streit zwischen Bürgern und durchmarschierenden Militärs zu vermeiden, wie aus früheren Stationierungen von französischen Soldaten in Hannover bekannt, erließen die Polizeikommissare am 16. Februar eine Polizeiverordnung: „nous avons défendu aux marchands et libraires de ne pas avoir [sic!] devant leurs fenêtres les portraits et figures des militaires de l'armée Britannique et Russienne, afin que cela ne donne pas d'occasion à un soldat ivre qui passoit, de casser les fenêtres oú de commettre d'autre excès". ${ }^{11}$ Obgleich Taberger keine englischen oder russischen, sondern französische Soldaten präsentierte, wurde ihm die Aufstellung des Sarges auf Grundlage dieser Verordnung zum Vorwurf gemacht.

Die Aufstellung mußte ,jedem Vorübergehenden - besonders den Militairs - auffallend seyn", schrieb Frömbling in seinem ersten Bericht. ${ }^{12}$ Taberger bestätigte ihn zum Teil in seiner Annahme, daß alle Passanten als potentielle Adressaten der Aufstellung gemeint waren, als er zu seiner Entlastung anführte, „die Leute [sollten] sehen ..., daß er auch solche Arbeit" mit Geschick erledigen könne. ${ }^{13}$

Unmittelbar nach der Überprüfung der Wahrhaftigkeit der Anzeige bezüglich des Sarges im Schaufenster Tabergers stellte Frömbling den Zinngießer in dessen Laden zur Rede. Das erste Argument, das er gegen Taberger vorbrachte, betraf den beleidigenden Charakter der Aufstellung, der „eine hinzielende Absicht nicht verkennen ließe“. ${ }^{14}$ Der Zinngießer betonte, er habe „keine Absicht zum Auffallen hierbei gehabt“, der Sarg gehe auf eine Bestellung zurück, und es sei Zufall gewesen, daß er den Sarg im Schaufenster mitten unter die französischen Soldaten gestellt habe. ${ }^{15}$ Daraufhin entschloß sich Frömbling, den Sarg zu beschlagnahmen, und beorderte Taberger zur näheren Befragung ins Polizeibüro. Dort übernahm noch am selben Tag sein Kollege Grahn die Vernehmung Tabergers. Er wurde erneut mit Deutlichkeit auf das Aussehen des Sarges und auf dessen Anordnung zwischen französischen Militärfiguren aufmerksam gemacht. Mitunter fiel als Beweis seiner bösen Absicht der Einwurf, daß „doch ein solcher Sarg grade kein Spielzeug sey, zu dem sich viele Käufer finden würden“, daß er also nicht aus Verkaufsgründen im Schaufenster ausgestellt worden sein könnte. ${ }^{16}$ Hie-

10 Vgl. Polizeibericht von Grahn und Frömbling an Bongars, 16. Febr. 1813, und Polizeibericht von Grahn und Frömbling an Bongars, 20. Febr. 1813, RNB, F 993 Arch. Westf., K. 13, N 7604-7687, hier $\mathrm{N}^{\circ} 7631$, 7632.

11 Polizeibericht von Grahn und Frömbling an Guntz, Generalpolizeikommissar in Braunschweig, 16. Febr. 1813, HStAH, Hann. 52, Nr. 812, B1. 179.

12 Abschrift des Polizeiprotokolls betr. den Zinngießer Taberger, 22. Febr. 1813, ebd., B1. 185f.

13 Ebd., B1. 181f.

14 Ebd., B1. 185f.

15 Ebd.

16 Abschrift der Polizeiprotokolle betr. den Zinngießer Taberger, 22. Febr. 1813, HStAH, Hann. 52, Nr. 812, B1. 181f. 
rauf wiederholte Taberger, daß der Sarg aufgrund einer Bestellung entstanden sei, und führte den Postkontrolleur Delarochette als Auftraggeber an. Erfreut über die eigene geleistete Arbeit, habe er den Sarg ausgestellt, um bekanntzumachen, daß er auch solche Arbeiten wie Beschläge und Griffe an Särgen anfertigen könne. Er beteuerte erneut, daß es Zufall gewesen sei, daß der Sarg vor den französischen Militärs einen Platz in seinem Schaufenster gefunden habe. „Er habe hierbey nicht die mindeste Neben-Idee gehabt" ${ }^{\text {". }}{ }^{17}$ Taberger schickte sich sogar zu seiner Verteidigung an, auf das Aussehen des Sarges einzugehen und detailliert zu beweisen, daß die Farbe der Beschläge nur weiß sein könne. Grahn blieb allerdings der Auffassung, „die Unzulänglichkeit seiner Entschuldigungsgründe und der hohe Grad von Unverstand und Leichtsinn, ... der in seiner Handlung liege“, seien auffällig. ${ }^{18}$ Taberger habe sich außerdem über die Verordnung vom 16. Februar hinweggesetzt, „die ihm erst vorkurzem von der Polizey aufgegeben sey, um Anordnungen zu vermeiden, alle unter der jetzigen Conjunctur von anstößige Gegenstande, vor den Fenstern wegzunehmen". ${ }^{19}$ Er habe sich auf jeden Fall eine „höchst strafbare Unvorsichtigkeit“ geleistet, so daß nicht allein der Sarg konfisziert bleibe, sondern auch der Polizeichef Bongars von dem Vergehen Tabergers in Kenntnis gesetzt werde.

Bei den folgenden Ermittlungen wurde Taberger der Falschaussage bezichtigt. Der Postkontrolleur Delarochette bestritt, den Auftrag zum fraglichen Sarg jemals gegeben zu haben. Die Polizeikommissare erblickten in der offensichtlichen Lüge Tabergers einen weiteren Beweis seiner bösen Absicht. Damit sei die „höchst strafbare Handlung" nicht mehr abzuweisen und seine Überführung nach Kassel zu eingehenderer Untersuchung begründet. Der bestürzte Taberger bekräftigte nochmals seine Unschuld. Als Entschuldigungsgrund führte er diesmal seine Naivität an, wo er doch „aus Freude darüber, dass ihm [der Sarg] so gut gerathen sey", ihn habe ausstellen wollen. Die Behauptung, daß Delarochette den Sarg bei ihm bestellt habe, sei ihm bloß „in der ersten Bestürzung" eingefallen - in der Hoffnung, so das Objekt schneller zurückzuerhalten. ${ }^{20}$

Beim überlieferten Quellenmaterial fällt insbesondere die Diskrepanz zwischen der unausgesprochenen bzw. ungenauen Beschuldigung durch die Polizeikommissare und der unerschütterlichen Bestimmtheit auf, mit der diese die Tat Tabergers als politisch verwerflich einstuften. Unablässig wird der Sarg in den Mittelpunkt der Beschuldigung und der Argumentation gerückt und hin und wieder mit Genauigkeit beschrieben, als ob seine Erscheinung von sich aus einen selbsterklärenden Charakter besitze. Erst in einem späteren Bericht in deutscher Sprache an den Generalpolizeikommissar Guntz wurde die pauschale Interpretation der Handlung Tabergers durch die Polizeikommissare in der französischen Zusammenfassung im Kopfteil geliefert: die Sarginszenierung sei „en allusion des évenements malheureux en Russie“ entstanden. ${ }^{21}$ Der Rußlandfeld-

17 Ebd.

18 Ebd.

19 Ebd.

20 Abschrift des Polizeiprotokolls betr. den Zinngießer Taberger, 2. März 1813, ebd., B1. 182f.

21 Bericht der Polizeikommissare Grahn und Frömbling an den Generalpolizeikommissar Guntz in Braunschweig, 4. März 1813, HStAH, Hann. 52, Nr. 812, B1. 180. 
zug Napoleons, der für seine Armee so vernichtend ausfiel, war ihrer Meinung nach gemeint. War der Sarg aber eine Anspielung auf den Untergang der französischen Herrschaft? Oder sollte er vielmehr eine Inszenierung von Napoleons Tod darstellen? War er als Todesnachricht oder als Prophezeiung gemeint? Bei der Interpretation der Sargausstellung durch die Polizeikommissare kann zunächst lediglich als sicher gelten, daß diese besorgt um das französische Militär waren, das gerade geschwächt vom Rußlandfeldzug zurückkehrte, und um dessen Interpretation der Tabergerschen Ausstellung. Sie befürchteten, das Militär könnte Anstoß nehmen an dem Sarg. Wollten sie mit der Konfiszierung vermeiden, daß der Sarg von den französischen Soldaten als Prophezeiung des eigenen sicheren Todes interpretiert werden könnte bzw. einen Entmutigungsfaktor in bezug auf den Krieg für sie darstellen könnte? Über den Kern ihrer Befürchtungen bleiben die Polizeikommissare in den Verhören mit Taberger und selbst in ihren Berichten an ihre Vorgesetzten ungenau. Es wird nicht explizit dargelegt, worauf die „böse Absicht“ Tabergers ihrer Ansicht nach hinauslaufen sollte.

Einige Stellen im Quellenmaterial weisen dennoch indirekt darauf hin, daß die maßgebliche Befürchtung der Polizeikommissare gewesen sein könnte, daß in der Sarginszenierung eine Anspielung auf den Tod Napoleons erkannt werden könnte. Worauf diese These im Quellenmaterial zur Affäre Taberger basiert und wie sie sich durch Hinzuziehung weiteren Quellenmaterials erhärten läßt, wird noch behandelt werden. Bevor diese zum Teil verdeckten Indizien entschüsselt werden, soll zur weiteren Einordnung und Kontextualisierung der Affäre Taberger ein Exkurs über den Umgang mit dem Tod in der westphälischen Gesellschaft unternommen werden.

\section{Zum Umgang der westphälischen Gesellschaft mit dem Tod}

War das Verhältnis zu Tod und Sterben in der westphälischen Gesellschaft so tabuisiert, daß Taberger mit seinem kleinen Sarg aus Blei derartiges Aufsehen erregen konnte? Im folgenden wird der Umgang mit dem Tod und mit Todesnachrichten anhand anderer zeitgenössischer Quellen untersucht, um den Kontext der Tabergerschen Sarginszenierung besser zu erschließen.

Daß die westphälische Polizei mit ihrer Konfiszierung des Sarges nicht zum ersten Mal in der kurzen Geschichte des Königreichs Westphalen in die Darstellung eines Begräbnisses eingriff, geht aus einer Einsicht der Polizeiarchive deutlich hervor. Bereits im März 1809 hatte die Polizei in Kassel ein Sargtuch nach einer Beerdigung beschlagnahmt und eine Untersuchung eingeleitet. Der Kasseler Polizeipräfekt schrieb über den Sachverhalt an den Polizeikommissar Kautz:

„Une Confrererie ... s'est servi hier à un enterrement d'un drap mortuaire, que j'ai l'honneur de vous faire remettre dans la présente, sur lequel sont brodés en lettre d'or, les mots K. Hessen. Ce qui veut dire Kuhr Hessen ou electorat de Hesse; j'ai du faire saisir cette emblême, d'un gouvernement qui n'existe plus et qui ne peut servir qu'à rappeller publiquement, un pouvoir qui n'existe plus dans ce pays cy. ... Vous remarqueréz aisement que la préposition Kuhr, qui n'existe plus aujourd' hui, que par l'initiale K. devant l'adjectif Hessen, qui précede le substantif, Con- 
frererie (Bruderschaft) était il n'y a pas longtemps écrite en toutes lettres, dont les premieres ont été recemment décousues, d'après les traces que vous verrés encore et vous reconnoitre facilement que c'est de l'ancienne finale $\mathrm{R}$. que l'on a fait l'initiale actuelle $\mathrm{K}^{\text {“.22 }}$

Mit dieser Mitteilung verknüpfte der Polizeipräfekt die Aufgabe, die Verantwortlichen ausfindig zu machen, wo doch „la confrerie ne pouvait certainement pas ignorer qu'elle ne vivait plus en Hesse, mais en Westphalie“. ${ }^{23}$ Die Affäre wurde jedoch nach wenigen Tagen zu den Akten gelegt, nachdem der Justizminister sich über die Beschlagnahme empört hatte und der Sargtuchbesitzer in Anwesenheit des Präfekten des Fuldadepartements die „mots inconvenants“ mit eigener Hand entfernt hatte. ${ }^{24}$

Das Sargtuch von Kassel aus dem Jahre 1809 erregte doppelten „Anstoß“: Die Polizei beanstandete zum einen das im Rahmen eines Begräbnisses stattgefundene versteckte Gedenken an das Kurfürstentum Hessen, das durch die westphälische Herrschaft abgelöst worden war. Der Justizminister Siméon störte sich zum anderen an dem Gedanken, daß die Polizei das Sargtuch möglicherweise mitten in der Prozession beschlagnahmt habe - was jedoch nicht der Fall gewesen war.

Auch weitere Polizeiberichte aus Hannover im März 1810 und aus Göttingen im Juli 1812 beweisen, daß Begräbnisse von der Polizei unter Beobachtung gestellt wurden, aus Furcht, sie könnten insbesondere Anlaß zur ungezügelten politischen Meinungsäußerung und zum nostalgischen Gedenken an die vormaligen Herrschaften bieten. ${ }^{25}$ Die Polizeikommissare befürchteten, daß bei Beerdigungen und Grabreden übersteigerte Emotionen der trauernden westphälischen Bürger hervorgerufen werden könnten, die zu Kritik an der westphälischen Herrschaft führen könnten. Daß die Polizeikommissare Frömbling und Grahn beim Anblick des kleinen Sarges im Schaufenster des Zinngießers Taberger im Februar 1813 diesem eine „böse Absicht“ unterstellten, überrascht vor diesem Hintergrund nicht weiter.

$\mathrm{Zu}$ den antifranzösischen Helden der napoleonischen Zeit zählte der Major Ferdinand von Schill, dessen Aktivitäten in Hinsicht auf den Umgang der Westphalen mit dem Tod einige Erkenntnisse ermöglichen. Im Frühjahr 1809 setzte er sich von Berlin $\mathrm{ab}$ und zog unter anderem durch das Königreich Westphalen, in der Hoffnung, die Bevölkerung gegen die ,Fremdherrschaft' aufzuwiegeln. Sein Unternehmen endete mit seiner Niederlage in Stralsund, wo er am 31. Mai 1809 selbst getötet wurde und die Franzosen über 550 Waffenbrüder seines Freikorps gefangennahmen. ${ }^{26}$ Einige

22 Schreiben des Polizeipräfekten von Kassel an den Polizeikommissar Kautz, 15. März 1809, GStAPK, V, HA., Rep. II., Abt. I., Nr. 2, Bd. 1.

23 Ebd.

24 Vgl. Schreiben des Polizeipräfekten von Kassel an den Justizminister Siméon, 16. März 1809, und Schreiben des Polizeipräfekten von Kassel an den Präfekten des Fuldadepartements, 18. März 1809, ebd.

25 Vgl. Rapport journalier de Police de la ville d'Hannovre du 30. Mars 1810, HStAH, Hann. 52, Nr. 3563; Schreiben vom Generalpolizeikommissar Mertens aus Göttingen an Bongars, 18. Juli 1812, RNB, F 993 Arch. Westf., K. 8, $\mathrm{N}^{\circ} 3723-3748$, hier $\mathrm{N}^{\circ} 3736$; ferner über das Verbot aller Beileidsäußerung in Braunschweig in Folge der Hinrichtung von Schill-Anhängern im Jahre 1809: Nagel, Kriegsbilder, S. 185.

26 Vgl. ebd., S. 174. 
Gefangene westphälischer Herkunft wurden vor ein Kriegsgericht gestellt und hingerichtet. Die Offiziere führte man in Gefangenschaft nach Wesel. Die Unteroffiziere und einfachen Soldaten wurden zum Galeerendienst nach Frankreich gebracht. ${ }^{27}$ Die Rezeption des Schillschen Feldzuges durch die zeitgenössische Gesellschaft erbringt nach genauerem Quellenvergleich unerwartete Erkenntnisse für den anstehenden Exkurs über den Stellenwert des Todes in der westphälischen Gesellschaft. Schill erfuhr nämlich nach seinem Tod ein Fortleben in der Phantasie und im Kollektivgedächtnis seiner Zeitgenossen.

Obgleich im Mai 1812 der Feldzug Schills mit seiner Niederlage und seinem Tod endgültig abgeschlossen war, warnte der Polizeichef Bongars die Generalpolizeikommissare in einem Rundschreiben vor einem „supplement probablement faux de la Gazette de Berlin, dans lequel supplement le major Schill et plusieurs officiers de son corps se recommandent au souvenir des habitans de la rive gauche de l'Elbe“. ${ }^{28}$ Im Juni darauf bestätigte ebenfalls der Generalpolizeikommissar Boehmer Bongars: „La malveillance fait même revivre le Colonel Schill'“ ${ }^{29}$ Nach Schills Tod soll außerdem, so der Zeitgenosse Friedrich Nagel, ein Gedicht, in dem Schill als „Geisterstimme ... selbstredend“ erschien, als Lied in Preußen und nicht zuletzt im Königreich Westphalen viel Anklang gefunden haben..$^{30}$

Diese Versuche, Schill im kollektiven Gedächtnis wiederzubeleben, waren nicht neu. Schon unmittelbar nach seinem Tod Ende Mai 1809 hatten mehrere Gerüchte von seiner leibhaftigen Wiederauferstehung gehandelt. Der Staatsgefangene Karl Christian von Gehren, Pfarrer zu Felsberg im Werradepartement, der wegen Teilnahme an den Unruhen im Zuge des Dörnbergschen Aufstands von 1809 festgenommen wurde, berichtet in seinen Memoiren über die Entstehung eines solchen Gerüchtes. Im Juni 1809 wurde er mit „48 Arrestanten, überwiesenen, verurtheilten, aneinander geketteten Insurgenten, österreichischen Ueberläufern, auch Schillianern" unter Begleitung von Gendarmen nach Mainz gebracht. ${ }^{31}$ Er schrieb über ihre Ankunft in Frankfurt am Main: „Wahrhaft brüderlich ... war unsere Behandlung in der Stadt Frankfurt am Meyn, die wir am neunzehnten Junius erreichten. Kurz vor der Stadt entspann sich zwischen mir, der ich eben neben den Wagen hergieng, und einem Franzosen, der daselbst garnisoniren mochte, folgendes Gespräch: ,N'est ce pas, Monsieur, (indem er auf meine Reisegefährten zeigte) ce sonst des Brigands?' Excusés, Monsieur, ce sont des Patriotes. ,Mais, ils sont donc des Prisonniers?' Tout à fait, ce sont des Prisonniers. ,Eh diable m'emporte, des Prisonniers, des Brigands - quelle différence?' Ah, mon citoien, croiez moi, aujourd'hui il y a beaucoup de Prisonniers, qui ne sont pad de [sic!] Brigands, comme il y a aussi beaucoup de Brigands, qui ne sont pas Prisonniers. Dieser Zusatz

27 Vgl. Bärsch, Ferdinand, S. 123f.; vgl. Nagel, Kriegsbilder, S. 176f.

28 Schreiben des Generalpolizeikommissars von Boehmer aus Göttingen an Bongars, 10. Mai 1812, RNB, F 993 Arch. Westf., K. 7, N 3649-3688, hier N 3671; vgl. Bericht von Bongars an den König Jérôme, 10. Mai 1812, RNB, F 993 Arch. Westf., K. 3, N³83-441.

29 Schreiben des Generalpolizeikommissars von Boehmer aus Göttingen an Bongars, 10. Juni 1812, RNB, F 993 Arch. Westf., K. 8, $\mathrm{N}^{\circ} 3689-3722$, hier $\mathrm{N}^{\circ} 3691$.

30 Nagel, Kriegsbilder, S. 172f.

31 von Gehren, Pfarrer 1816, S. 88; vgl. ferner Nagel, Kriegsbilder, S. 176ff., 182. 
geschah nicht ohne Bitterkeit. Der Soldat machte also große Augen, maß mich von Kopf bis zu Fuß, überschaute die ganze Karavane, und eilte nun zum Thore hinein, ... nicht ohne Besorgniß sah ich, daß der französische Soldat mit dem Wachehabenden Officier, ... lebhaft sich unterredete. ... Inzwischen, wir fuhren ruhig durch das Thor und vor der Wache vorbey. Der Franzose, den ich immer im Auge behielt, begleitete uns und war stets neben meinem Wagen. Er redete mit mehreren auf der Straße deutsch. Mit jedem Schritte vermehrte sich unsere Begleitung, die uns neugierig betrachtete. Endlich erreichten wir die Zeile, und hier wurde das Gedränge so groß, daß unsere Wagen still halten mußten. In einer Stadt, wie Frankfurt, wo man sonst nicht so neugierig ist, wie in kleinen Städten, fiel mir dieß sehr auf. ,Wissen Sie wohl' sagte Einer der Gensdarmen, der sich mir näherte, ,daß man Sie sämtlich für Schillianer hält und daß ein Franzose gesagt haben soll: Schill selbst sey mitten unter Ihnen?' Möglicher Weise hatte meine Unterredung mit jenem Soldaten Anlaß zu dieser Meynung gegeben. Einer aus unserer Mitte, ein ausnehmend stärker, mit fürchterlichem Backenbarte versehener, Mann, ein Knopfmacher von Homberg, wurde bald für Schill gehalten und die den Wagen umzingelnde Menge gab durch Geld und andere Geschenke, die sie unter unsere Insurgenten vertheilte, deutlich zu erkennen, wie sie über Schill dachte“.32

Durch Gehrens Erzählung wird die Entstehung eines Gerüchts anschaulich und eine zentrale Grundbedingung für sein Entstehen deutlich: Die Frankfurter waren gewillt, Schill am Leben zu glauben und huldigten bewußt oder unbewußt in der Person eines Knopfmachers dem Gedächtnis des gefallenen Majors. Ihre Hingabe an das Gerücht führte zu Geldspenden und materiellen Geschenken. Die Nachricht vom Einzug der „Schillianer" in Frankfurt am Main, worunter selbst Schill vertreten sein sollte, gewann sicherlich insbesondere an Glaubwürdigkeit, da ausgerechnet ein Franzose, der der deutschen Sprache mächtig war, für die Verbreitung der Nachricht sorgte. Möglicherweise wurde das Gerücht von Kritikern der Zeit freudig weitergetragen.

Wenn im Juni 1809 und erneut im Jahre 1812 geglaubt wurde, Schill sei noch am Leben, so lassen auch andere Todes- und Lebensnachrichten vermuten, daß die Grenze zwischen Tod und Leben besonders im Fall von Kriegshelden und Feldherren dünn war. Im Fall der sogenannten ,schwarzen“ Husaren des Herzogs von BraunschweigOels, der ebenfalls im Juli 1809 einen Zug durch das Königreich Westphalen mit der gleichen Motivation wie Schill vor ihm unternahm, sorgte schon zu ihren Lebzeiten ihre Erscheinung in Totengestalt für Aufsehen. Polizeiberichte aus dem Großherzogtum Berg an den französischen Polizeiminister in Paris wissen zu berichten:

„J'appèle M. le Duc de Brunswick un brigand, parcequ'il a commis les excès les plus cruels à Leipzick et à Halle, et que sous ce misserable rapport il s'est placé fort audessous de Schill. Il s'est placé surtout par le ridicule. Il intitule ses bandes, Légion de vengeance; il habille ses brigands en noir; il les chamare d'ossemens humains et de têtes de mort brodés en blanc; il leur fait prêter des serments lugubres““.33

32 von Gehren, Pfarrer 1816, S. 105-108.

33 Rapport de la $1^{\text {re }}$ quinzaine du mois d'aout courant, Rapport du 11 aout 1809 , Rubrique „Westphalie“, AN Paris, AF ${ }^{\mathrm{IV}}$ 1839. Bei einem Vergleich zwischen dem Schill'schen Freikorps und den „schwarzen Husaren" des Herzogs von Braunschweig hinsichtlich des Umgangs der Westphalen mit Tod und Leben fällt 
Im Kontext des Krieges verbreiteten sich im allgemeinen Todes- und Lebensnachrichten, ohne daß die Möglichkeit bestand, unmittelbar ihren Wahrheitsgehalt zu überprüfen. So berichteten Polizeiagenten häufiger von solchen öffentlich verbreiteten Nachrichten, wobei sie nicht in der Lage waren, auszumachen, ob es sich lediglich um Gerüchte handelte oder ob diese der Wahrheit entsprachen. ${ }^{34}$ Die beschönigende Kriegsberichterstattung in den öffentlichen Blättern wird dazu beigetragen haben, daß die Nachrichten von der Front nicht für sicher gehalten wurden. Ein Zeitgenosse schrieb über die französischen Meldungen vom Krieg: „Die Franzosen pflegten in ihren Schlachtenberichten die Verluste der Gegner, um ihre Tapferkeit in eine helle strahlendes Licht zu stellen, bis ins Unglaubliche zu übertreiben; die ihrigen hingegen so geringe anzugeben, daß man hätte glauben sollen, ihre Feinde hätten mit Erbsen geschossen und mit Fuchslunten gefochten“. ${ }^{35}$

Die Schreckensnachrichten vom Rußlandfeldzug breiteten sich jedoch dessen ungeachtet aus. So wurde Anfang Februar 1813 in Göttingen bekannt, daß der Perückenmacher Rapper aus Northeim gesagt haben sollte: „que les restes de neuf Regiments westphaliens, ensemble 25 hommes, avoient passé par Northeim “. ${ }^{36}$ Der Kandidat der Theologie Grosse aus Niedenstein im Elbedepartement habe sich ebenfalls Anfang Februar erdreistet, „unter der Zeitung ..., worinn bemerkt war, daß Napoleon viele Truppen marchiren lässt, [in roter Schrift den Kommentar:] (wann der Schnee in Rußland wiederum aufthauet)“ zu setzen. ${ }^{37}$ Dies war eine sarkastische Anspielung darauf, daß die napoleonische Armee größtenteils unter dem Schnee in Rußland liege und erst mit dem Frühling wieder auftauchen werde. Grosses ironischer Umgang mit dem Massensterben von Soldaten der Grande Armée und ihrer Verbündeten in Rußland und seine kritische Rezension der westphälischen Kriegsberichtserstattung durch Randbemerkungen in roter Schrift in einem Exemplar des „Monitors“ verursachten seine Verhaftung und Überführung ins Kastell von Kassel. ${ }^{38}$ Die harten polizeilichen Maßnahmen gegenüber Verbreitern von Todesnachrichten aus Rußland hatten einen klaren Hintergrund. Es wurde befürchtet, daß derartige Nachrichten die neuen Rekru-

die Umkehrung des Motivs auf: Wurde Schill nach seinem Tod mehrfach wiederbelebt, so gebärdeten sich die Husaren des Herzogs von Braunschweig-Oels als Rächer und drückten möglicherweise mit ihrer Verkleidung den ersehnten Tod der Franzosen aus.

${ }^{34}$ Der Polizeiagent von Baerll berichtete mit einem großen Maß Ungewißheit in seinem Bericht an von Schalck über den tot geglaubten General Vandamme und den noch am Leben seienden General Moreau: vgl. Agentenbericht von Vb [= von Baerll] an Schalck, 15. Sept. 1813, RNB, F 993 Arch. Westf., K. 9, N 4347-4351. Der Polizeiagent Heiliger berichtete dem Generalpolizeikommissar Guntz das Gerücht vom Attentat auf Bernadotte, den König von Schweden, im November 1812: vgl. Agentenbericht von Heiliger an Guntz, 19. Nov. 1812, HStAH, Hann. 52, Nr. 812, B1. 140.

35 Nagel, Kriegsbilder, S. 169.

36 Bericht des Generalpolizeikommissars Mertens in Göttingen an Bongars, 13. Febr. 1813, RNB, F 993 Arch. Westf., K. 14, $\mathrm{N}^{\circ}$ 8041-8158.

37 Agentenbericht von C [= Cerfy], 18. Febr. 1813, RNB, F 993 Arch. Westf., K. 16, N 9760-9796.

38 Vgl. Affäre Grosse, RNB, F 993 Arch. Westf., K. 21, N 13155-13179; ferner Schreiben von Bongars an den Maire in Niedenstein, 8. Febr. 1813, RNB, F 993 Arch. Westf., $N^{\circ} 13850$; Schreiben von Bongars an den Kantonmaire in Niedenstein $\mathrm{N}^{\circ} 273$ vom 8. Febr. 1813, ebd.; Schreiben von Bongars an den Friedensrichter Pfeiffer in Niedenstein, 8. Febr. 1813, ebd.; Schreiben von Bongars an den Kastellkommandant in Kassel, 14. Febr. 1813, ebd.; vgl. ferner RNB, F 993 Arch. Westf., № 13852, Eintrag Nº 103. 
ten entmutigen würden. So wußte der Polizeiagent Eskuchen über die Stimmung bei den neuen westphälischen Soldaten im Mai 1813 zu berichten: „L'esprit qui regne entre les Soldats Westphaliens est encore assez mauvoix; ils sont remplis de peur de perdre leur vie, et ce sont eux-même qui se communiquent ces sentiments; on entend souvant les [sic !] parler de la sorte, cammerades faisons bonne chère, car l'année passé nous n'existerons plus; c'est les Russes qui nous aurons tués“. ${ }^{39}$ Die Todesnachrichten erzeugten unmittelbar Todesangst.

Daß die kursierenden Todesnachrichten im Februar 1813 im allgemeinen für glaubwürdig gehalten wurden, zeigt sich auch an der Affäre Fraengel vom Winter 1812. Im November 1812 meldete Bongars dem Generalpolizeikommissar in Halberstadt: „un individu qui s'occupait d'écrire aux gens crédule [sic !] qui ont des enfans à l'armée, pour les tromper sur le sort de ces derniers, et qu'en outre pour prouver ses absurdités, il leur envoyait des Extraits mortuaires qu'il fabriquait à cet effet" ${ }^{40}$ Die Motivation des verhafteten Fraengel war, den von der Todesnachricht ihrer Nächsten bestürzten Verwandten unter Berufung auf vermeintliche Schulden der angeblich Verstorbenen Geld zu entlocken. Aber nicht allein Fraengel kam auf den Gedanken, mit den Todesnachrichten ein Geschäft zu machen: Auch ein Mann namens Trettstedt wurde wegen der Herstellung von falschen Totenscheinen und des Betrugs von verzweifelten Familien im Dezember 1812 verhaftet. ${ }^{41}$ Die allgemein verbreitete Angst vor dem Krieg und seinen Folgen machte den Betrug mit Totenscheinen möglich.

In den Wirren der Nachrichten über Tod und Leben von Soldaten und Kriegsherren wird es wiederum durchaus denkbar, daß Taberger mit dem kleinen Sarg unter anderem auf den Tod Napoleons anspielen wollte. Politische Gegner sterben und vaterländische Helden wiederauferstehen zu lassen, scheint durchaus gängig gewesen zu sein. Aufgezeigt wurde dies u.a. anhand der Gerüchte über die Ankunft Schills in Frankfurt am Main nach dessen Tod, der Lieder und der Pamphlete, wie im Fall des gefälschten Supplements der Berliner Gazette, das Schill noch 1812 für am Leben erklärte. Über die vielen angeblichen Attentate auf Napoleon und seine Familie soll im folgenden noch berichtet werden, damit diese in die Interpretation der Tabergerschen Sarginszenierung einfließen können.

In der Nacht vom 23. auf den 24. März 1813 wurden einige Kasseler Bürger der Rue des Orphelins aus dem Schlaf gerissen. Der Referendar der Oberrechenkammer Kaulwell fühlte sich nämlich dringend genötigt, ein Geständnis vor den von seinem Lärm herbeigerufenen Gendarmen Meise und Lübbers abzulegen:

39 Agentenbericht des Polizeiagenten E [= Eskuchen] N 68, 18. Mai 1813, RNB, F 993 Arch. Westf., K. 10, $\mathrm{N}^{\circ} 5041-5085$, hier $\mathrm{N}^{\circ} 5055$.

40 Schreiben von Bongars an Moisez, Generalpolizeikommissar in Halberstadt $\mathrm{N}^{\circ}$ 2894, 25. Nov. 1812, GStAPK, V, HA., Rep. II., Abt. I., Nr. 4, Bd. 5; vgl. Schreiben von Bongars an Moisez N 2906, 25. Nov. 1812 , ebd.

41 Vgl. Schreiben von Bongars N 3187, 29. Dez. 1812, GStAPK, V, HA., Rep. II., Abt. I., Nr. 4, Bd. 5; Schreiben von Bongars an den Generalpolizeikommissar Moisez in Halberstadt, 29. Dez. 1812, RNB, F 993 Arch. Westf., $\mathrm{N}^{\circ} 13848$; zu weiteren Vorfällen: Rundschreiben von Bongars an die Präfekten, 25. Jan. 1813, GStAPK, V, HA., Rep. II., Abt. I, Nr. 4, Bd. 5; LHASAW, B 18 II. 123. II a, B1. 262 f. 
„Ich bin unglücklich, ich habe ein Verbrechen begangen, Sie müssen mich arretiren, weil ich den festen Vorsatz hatte und schon lange damit umgegangen bin den König von Westphalen zu ermorden, ich habe stets versteckte Waffen zu diesem Zweck bei mir getragen und mit diesem Gedanken lange schwanger gegangen; um gerade jetzt will ich sterben, da mein armes Vaterland wieder in Gefahr ist“". ${ }^{42}$

Das Staatsverbrechen Kaulwells stellte sich jedoch bei näherer Untersuchung als recht harmlos dar, denn dieser habe „sich aus wahrscheinlicher Abwesenheit des Verstandes selbst des Verbrechens angeklagt“, wie die Gendarmen feststellten. ${ }^{43}$ Kaulwell wurde letztlich für labil und psychisch krank erklärt und von seiner selbst beteuerten Schuld freigesprochen. Die Fixierung Kaulwells auf das schwerste Verbrechen, den „crime lèse-majesté“, spricht für die allgemeine Faszination, die ein Attentat auf den König Jérôme in der westphälischen Gesellschaft hervorzurufen in der Lage war.

Nicht weniger Aufsehen erregte ein vermeintliches Attentat auf Napoleon selbst. Im Winter 1809 war der Domherr von Münster, Wilhelm von der Lippe, von der Polizei belangt worden, weil er Gerüchte über den Tod Napoleons verbreitet hatte. ${ }^{44} \mathrm{Im}$ Kontext des Rußlandfeldzugs ging auch im Januar 1813 in der Form von „lügenhaften Zetteln", diesmal im Halleschen Waisenhaus und in der Gegend von Halle, die Nachricht von Napoleons Tod um. ${ }^{45}$ Wie aus den Ermittlungen hervorging, waren die aufrührerischen Pamphlete, „qui portent les mots Bonaparte est todt! Auf!“, von den Schülern Eppner und Schulze angefertigt worden. ${ }^{46}$ Für ihre antifranzösischen Zettel wurden sie am 14. Februar in das Kasseler Kastell gebracht und erst Anfang Juli wieder freigelassen. Anfang April 1813 wurde im Rahmen eines Verhörs Caspar Simmer aus Altmorschen im Werradepartement, der unter anderem beschuldigt wurde, die westphälischen Untertanen in seiner Gegend zur Verweigerung ihrer Steuerabgaben anzustiften, ebenfalls wegen einer von ihm zuvor verbreiteten Nachricht zur Rechenschaft gezogen:

„Ist von ihnen nicht auch folgende Geschichte, die sich mit dem französischen Kaiser zugetragen haben soll, erzählt worden: ,der Kaiser wäre ja schon geflüchtet, die Russen hätten ihn beinahe gefangen genommen, indem derselbe so im Gedränge gewesen wäre, daß ein Kosacke in dem Wahn den Kaiser zu erstechen, einen seiner Generäle an seiner Seite gestochen habe: überhaupt hätte der Kaiser sich nur dadurch gerettet, deßen [daß er?] sich in Sachsen auf die Post gesetzt habe'“.47

42 Protokoll der Königlichen Gendarmerie über die Arrestation des Cammer Referendaire H. Friedrich Kaulwell, 23. März 1813, RNB, F 993 Arch. Westf., K. 16, N 10268-10346 (darin: N 10334-10339 Affäre Kaulwell, hier $\mathrm{N}^{\circ} 10338$ ).

43 Ebd.

44 Vgl. Dossier de Police October - Décember 1809, AN Paris, AFIV 1839 B.

45 Schreiben des Unterpräfekten Piautaz an Bongars, 27. Jan. 1813, RNB, F 993 Arch. Westf., K. 13, N $7725-$ 7761 , hier $\mathrm{N}^{\circ} 7758$.

46 Einträge $\mathrm{N}^{\circ} 104$ und $\mathrm{N}^{\circ} 105$, RNB, F 993 Arch. Westf., Registre des personnes arrêtées ... depuis le 1811, 1812, 1813; vgl. auch RNB, F 993 Arch. Westf., K. 21, N 13239-13254; Schreiben $N^{\circ} 358-359$, 366 und 371, RNB, F 993 Arch. Westf., $N^{\circ} 13850$.

47 Verhörprotokoll von Caspar Simmer in Kassel, 5. April 1813, RNB, F 993 Arch. Westf., K. 21, № 13084 13108 , hier $\mathrm{N}^{\circ} 13096$. 
Im Zeitraum von Juni bis Juli 1813 ging erneut ein Gerücht um, wonach „der französische Kayser von einem seiner Generäle durch einen pistolen Schuß, tötlich am Kinn plesirte worten" sei. ${ }^{48}$ Als erste berichtete die Polizeiagentin Gall-Bessalié aus Kassel am 23. Juni darüber:

„Der Sargent Vaf ... hat in einem hisigen [burgerhaus] volgende Begebenheit erzelt, die er von jemanden der aus Dresden kam, gehort haben will, Nemlich der Keiser Napoleon habe einem Genral mit die unter seinem Comendo stehende Truppen zu einer afairé beordert, der Genral habe ihm dagegen Vorstelungen gemacht und behauptet er habe dafür zu wenig Leite, das würde doch nichts helfen, der Keiser widerholte seinen gegebene Befehl, und es entstand ein disput, der Genral in der hitze schoß nach dem Keiser und traf ihm in [Hirn], und eine kleine Verletzung am Hals, der Genral vermuhtlich sein Verbrechen einsehand, habe sich selbst erschoBen". 49

Ob Kinn oder Hals, die verschiedenen Varianten des Gerüchts waren sich darüber einig, daß es sich um einen gefährlichen Kopfschuß und damit um den Kopf und die Krone des Kaisers handelte. Der Polizeiagent Cerfy berichtete am 29. Juni aus einem Kasseler Wirtshaus „von dem falschen Gerücht welches hier verbreitet wurde, nämlich daß Napoleon von seinem eigenen Général durch einen Pistohlen Schuß tödlich plessirt wurde".$^{50}$ Gall-Bessalié meldete nochmals am 1. Juli:

„Am Tische des Statsrats von Schulters wurde vor einigen Tagen, über das selbe Merchen gesprochen, was ich Ihnen bereits vor 12 Tagen schon anzeigte, nemlich das der Keiser habe einem Marschal beordert, mit seinem Truppen nach Berlin zu gehen, der Marschal widerriht, es werde nichts hulfen, er habe zu wenig leite, sie verden aus aufgeopfert, der Keiser wider holte seine Ordre mit dem Zusatz faite tout souite, und als Ihm der Marischal nochmals widersprach, habe der Keiser zu erst nach ihm geschoßen, der Marschal drikte eine pistole auf dem Keiser ab, wo die Kugel in der Gegend der Nase herein und am Kinn heraus kam, und schoß die zweite pistole auf sich selbst ab". .51

Die Information wurde von Mal zu Mal genauer, nun war der Kopfschuß bei der Nase zugefügt worden und wieder am Kinn ausgeschieden, auch hieß es in der zweiten Version der Polizeiagentin Gall-Bessalié, Napoleon habe zuerst geschossen. Das Gerücht nahm offenbar seinen Lauf auch außerhalb von Kassel. So warnte Bongars den Unterpräfekten von Hersfeld:

48 Agentenbericht des Polizeiagenten C [= Cerfy] an Bongars, 3. Juli 1813, RNB, F 993 Arch. Westf., K. 19, $\mathrm{N}^{\circ} 11919-12074$, hier $\mathrm{N}^{\circ} 12016$.

49 Agentenbericht der Polizeiagentin G.-B. [= Gall-Bessalié] an Bongars, 23. Juni 1813, RNB, F 993 Arch. Westf., K. 10, $\mathrm{N}^{\circ}$ 5030-5040, hier $\mathrm{N}^{\circ} 5040$.

50 Agentenbericht des Polizeiagenten C [= Cerfy] an Bongars, 29. Juni 1813, RNB, F 993 Arch. Westf., K. $10, \mathrm{~N}^{\circ} 5086-5125$, hier $\mathrm{N}^{\circ} 5124$.

51 Agentenbericht der Polizeiagentin G.-B. [= Gall-Bessalié] an Bongars, 1. Juli 1813, RNB, F 993 Arch. Westf., K. 19, $\mathrm{N}^{\circ} 11919-12074$, hier $\mathrm{N}^{\circ} 12066$. 
„Il m'est revenu ... que le Sr. Strutz, Procureur prés le tribunal de lere instance seant à Hersfeld a dit que des connoissances qu'il à Cassel lui ont communiqué pour nouvelle: 'que S.M. l'Empereur et Roi était blessé au menton par suite d'un différent qu'il aurait eu avec un de ses Généraux en chef sur le quel S.M. Impériale aurait tiré un coup de pistolét; et le Gal. ayant riposté il aurait atteint l'Empereur au menton, la balle serait glissée derniere le col, sans qu'on ait pu encore la retirer etc. etc'“.52

Bei dieser Nachricht von der lebensgefährlichen Verletzung des Kaisers wird deutlich, daß im Kontext des Jahres 1813 selbst aus den Rängen der napoleonischen Armee Attentate auf Napoleon für wahrscheinlich erschienen. Je mehr die napoleonische Eroberungspolitik zum Scheitern verurteilt schien, desto mehr Gerüchte über seinen gewaltsamen Tod verbreiteten sich. Das Fehlschlagen des Rußlandfeldzugs stellte den Ruf von Napoleons Unbesiegbarkeit in Frage. ${ }^{53}$ Angesichts der vielen Gerüchte über den Tod Napoleons läßt sich eine Verbindung zur Sarginszenierung von Taberger vermuten: Wollte dieser vielleicht den Kaiser für tot erklären, obgleich zu diesem Zeitpunkt Napoleon bereits wieder in Paris eingetroffen war?

\section{Der Sarg, die Mamelucken und das „Escadron sacré“ im Schaufenster des Zinngießers Taberger}

Vor dem Hintergrund des Verhältnisses vieler Westphalen zum Tod im allgemeinen und zu demjenigen Napoleons im besonderen soll die Interpretation der Sarginszenierung durch die Hannoverschen Polizeikommissare erneut analysiert werden. Eine Rückkehr zu der These, die aus der textimmanenten Interpretation des Quellenmaterials zur Affäre Taberger herausgebildet wurde, ist geboten: Stellte in den Augen der Hannoverschen Polizei der Sarg ein Sinnbild für Napoleons Tod dar? Drei Indizien führen zu einer präziseren Aussage.

Wie schon erwähnt, erschien der schwarze Sarg in den Augen der Polizeikommissare ganz besonders verdächtig, als sie beim genaueren Hinschauen die französischen Nationalfarben an ihm entdeckten: der „Sarg, an dem sich vorn und hinten ein blaues und rothes Schild mit weißer Einfassung befinden ". ${ }^{54}$ Der inszenierte Tod betraf offenbar unmittelbar einen Angehörigen der Grande Armee, wenn nicht die Armee als ganzes. Nach den Angaben der Polizeikommissare war der Sarg außerdem von „französische[n] Officiere[n] zu Pferde nebst 2 Mameluken, welches die hiesige Escadron vorstellen konnte“, umstellt - möglicherweise ein zweites Indiz in Richtung der oben aufgestellten These ${ }^{55}$ Die Erwähnung der Mameluken stellte einen unmittelbaren Bezug

52 Schreiben $\mathrm{N}^{\circ} 1730$ von Bongars an den Unterpräfekten in Hersfeld, 1. Juli 1813, RNB, F 993 Arch. Westf., $\mathrm{N}^{\circ} 13851,1$.

53 Vgl. Schmidt, Kriegsgott, S. 168, $173 \mathrm{f}$.

54 Abschrift der Polizeiprotokolle betr. den Zinngießer Taberger, 22. Febr. 1813, HStAH, Hann. 52, Nr. 812, B1. 181f.

55 Ebd., B1. $185 f$. 
zur berühmten kaiserlichen Garde her oder sogar zu Rustan, dem Diener Napoleons, der ihn auf Tritt und Schritt seit seiner Rückkehr aus Ägypten begleitete.

In den Memoiren des Pastors Nagel aus Halberstadt werden die Mameluken und Rustan mehrfach erwähnt. Als Nagel über die französische Reiterei und die Nationalgarde schreibt, kommt er auf sie zu sprechen:

„Noch viel mehr Aufsehen erregten jedoch die sogenannten Mamelucken, aus deren Mitte jener Rustan hervorgezogen sein mogte, der Leibmameluck, welchen wir später mehrmals auf dem Kutschbocke des Reisewagens gesehen haben, in dem der Kaiser fuhr. Nicht Alle waren jedoch so tiefbraun von Angesicht wie dieser. Man bemerkte Schattirung der Farben vom Gelb der Südeuropäer bis zur Olivenfarbe des Malaien, jedoch durchgehends morgenländische Gesichtsbildung und ein Colorit welches ebenfalls seine Abkunft vom Sonnenaufgang her verrieth. Es waren schöne Leute von schlankem Wuchse, völlig orientalisch uniformirt und gerüstet bis auf Sattel, Zaum und breite, türkische Steigbügel““. ${ }^{56}$

Diese Ausführungen machen deutlich, daß das Fremdländische der Mameluken, die unter den Reitern der Kaiserlichen Garde eine Kompanie bildeten, bei Truppendurchmärschen im Königreich Westphalen große Beachtung fand. Daß Rustan als der „Leibmameluck“ Teil der napoleonischen Legende zu Lebzeiten des Kaisers wurde, wird auch in der Forschungsliteratur bestätigt. ${ }^{57}$ Nagels Memoiren erwähnen eine weitere Erscheinung Rustans, als sich der Pastor Mitte September 1808 zur Messezeit in Frankfurt am Main aufhielt:

„... eine dichtverschlossene, mit vier Rappen bespannte Reisekutsche ... rasselte [über den Messeplatz bei uns vorüber]. [Napoleon] saß still und unbemerkt im Hintergrunde der Carosse ... Man ward nichts von ihm gewahr; Rustan jedoch, der Mamelucke, welcher den Bocksitz zierte, zog durch seine finstere Hautfarbe und die morgenländischen Gesichtszüge, in Verbindung mit dem gleichfalls orientalischen Habite, die Aufmerksamkeit auf sich und verrieth, daß sein kaiserlicher Gebieter nahe sei“ ${ }^{.58}$

Wenn bereits im Jahre 1808 in Frankfurt am Main die Anwesenheit des Mameluken Rustan für Nagel als Hinweis diente, Napoleon in einer Reisekutsche auf dem Weg zum Erfurter Fürstenkongreß zu vermuten, so könnte im späten Jahr des napoleonischen Kaiserreichs 1813 die Hervorhebung der Mameluken in der Beschreibung der Sarginszenierung durch die Polizeikommissare durchaus ein Indiz bilden für ihre Interpretation der Aufstellung der Figuren als Begräbnis des Kaisers. Der Mameluk Rustan war den Zeitgenossen ein konkreter Hinweis auf die Nähe des Kaisers. Dies erklärt vielleicht die unausgesprochene Beschuldigung Tabergers durch die Polizeikommissare in den Verhören. Sie versteckten zwar ihre Entrüstung über seine Sarginszenierung nicht, jedoch schreckten sie offenbar davor zurück, das Unmögliche an dieser Inszenierung klar zu benennen.

\footnotetext{
56 Nagel, Kriegsbilder, S. 48f.

57 Vgl. Pigeard, Dictionnaire, S. 386, 518.

58 Nagel, Kriegsbilder, S. 82f.
} 
Ihre Verschwiegenheit über den hintergründigen Sinn der Sarginszenierung könnte als Verhörstrategie gewertet werden. So versuchten sie möglicherweise, Taberger zu einer eindeutigen Aussage zu nötigen und eine eigene Deutung seiner Handlung aus ihm herauszulocken. Wenn die Polizeikommissare ihre Deutung der Sarginszenierung nicht explizit benannten, könnte es auch daran gelegen haben, daß sie sich scheuten, den Tod von „Napoleon l'immortel“ auszusprechen. ${ }^{59}$ Das Unsagbare, die Nachricht vom Umkommen des unsterblichen Napoleons im Rußlandfeldzug, in eigener Formulierung in das Verhörprotokoll oder in ihre Berichte aufzunehmen, unterließen sie letztlich. Sie beließen es bei Anspielungen, vielleicht auch, weil sie befürchteten, von ihren Vorgesetzten als Träger der Nachricht mitbeschuldigt zu werden.

In einem Bericht an den Polizeichef Bongars tritt die Aussage über die Sarginszenierung Tabergers jedoch sehr deutlich zutage. Dies ist auch das dritte Indiz, das dafür spricht, daß die Polizeikommissare den Sarg als Anspielung auf den Tod des französischen Kaisers verstanden. „Taberger ... a eu l'imprudence de mettre entre les figures d'étain, qui réprésentent des militaires françois et l'escadron sacré, un petit cercueil d'étain, où il y a de l'un côté et de l'autre un petit bouclier rouge et bleu avec des extrémités blanches“ ${ }^{60}$ Der „Escadron sacré“ ist ein konkreter Hinweis auf eine Besonderheit des Rußlandfeldzugs. Im 29. Bulletin der Grande Armee aus Molodetschno vom 3. Dezember 1812 wurde der „Escadron sacré“ zum ersten Mal erwähnt: „Notre cavalerie était tellement démontée que l'on a dû réunir les officiers auxquels il restait un cheval pour en former quatre compagnies de 150 hommes chacune. Les généraux y faisaient les fonctions de capitaine, et les colonels celles de sous-officiers. Cet escadron sacré, commandé par le général Grouchy, et sous les ordres du roi de Naples, ne perdait pas de vue l'Empereur dans tous ses mouvements" ${ }^{61}$ Bei der Rückkehr vom Rußlandfeldzug wurde die Sicherheit Napoleons zu einem zentralen Problem. Um die Gefahr einer Gefangennahme oder des Todes zu verringern, konstituierte man eine außerordentliche Garde zum Schutz des Kaisers, die von Joachim Murat geleitet wurde..$^{62}$

Diese drei Indizien - die französischen Nationalfarben an der Sargfassung, die Mameluken und das „Escadron sacré“ um den Sarg - unterstützen die anfangs aufgeworfene These, daß die Polizeikommissare in der Sarginszenierung einen Hinweis oder eine Prophezeiung des Todes Napoleons erblickten.

59 Anstellungsgesuch von Lombard an König Jérôme von Westphalen, 1. Febr. 1808, GStAPK, V, HA., Rep. VIII., I. B, Nr. 24. Vgl. ferner zur zeitgenössischen Auffassung von Napoleon als „Kriegsgott“: Carl Philipp Gottfried von Clausewitz, zitiert nach Schmidt, Kriegsgott, S. 167; zur allgemeinen Faszination des ,übermenschlichen' Napoleon: Becker, Zeiterfahrungen, S. 85.

60 Polizeibericht der Polizeikommissare Grahn und Frömbling an Bongars, 23. Febr. 1813, RNB, F 993 Arch. Westf., K. 13, $\mathrm{N}^{\circ} 7604-7687$, hier $\mathrm{N}^{\circ} 7637$.

61 Zitiert nach Pigeard, Dictionnaire, S. 243 (Artikel „Escadron sacré").

62 Vgl. ebd. 


\section{Tabergers Perspektive und die Wahrnehmung des Sarges durch die Hannoveraner}

Die Konsequenzen aus dieser Interpretation für Taberger hielten sich letztlich in Grenzen. An sich waren die Polizeikommissare geneigt, ihn mit einer scharfen Zurechtweisung gehen zu lassen, allerdings gefiel Bongars diese Milde, ihr „esprit de tolérance bien répréhensible" nicht. ${ }^{63}$ Er wünschte vielmehr, ein Exempel zu statuieren und ordnete an, Taberger und seinen kleinen Sarg nach Kassel zu schicken. ${ }^{64}$ Denn „il y a ici une intention equivoque qui mérite une punition exemplaire" ${ }^{65}$ Sein Wille scheiterte jedoch daran, daß Taberger krank wurde. Die Polizeikommissare unterrichteten Bongars: „Cet individu a été tellement effrayé qu'il en est tombé malade au point de ne pouvoir pas étre transporté; c'est du moins ce que declare le medecin dans le certificat que vous m'avez transmis". ${ }^{66}$ Taberger richtete sich mit einem Gesuch selbst an Bongars, um Nachsehen zu erbitten. Bongars zweifelte jedoch an Tabergers Krankheit und befahl, daß dieser, sobald wiederhergestellt, nach Kassel abtransportiert werde, solange dies nicht möglich sei, unter Hausarrest stehe und die Überwachung durch einen Gendarmen selbst tragen müsse.$^{67}$ Letztlich gab Bongars die Idee auf, an Taberger ein Exempel zu statuieren. Am 16. März schrieb er nach Hannover:

„Il m'a été adressé de vives sollicitations à l'égard de ce prevenu, voulant y avoir quelques égards, je vous autorise à le remettre en liberté à charge de demeurer sous votre surveillance spéciale jusqu'à ce qu'il vous ait déclaré le motif qui l'a porté à placer le cercueil en question au milieu de militaire français““. 68

Diese Entscheidung Bongars' ist das letzte überlieferte Dokument im Dossier Taberger, der nicht mehr in den Akten der politischen Polizei auftauchen sollte. Bongars wies in diesem letzten Schreiben zum Fall Taberger darauf hin, daß die Ermittlungen zur in seinen Augen „intention equivoque“ Tabergers vom Betroffenen selbst noch keine eigene Deutung seiner Tat hervorgebracht hätten.

Tabergers Intention zu rekonstruieren ist auf der Grundlage der Verhörprotokolle unmöglich - hier spielt erneut die Autoritätsfrage eine Rolle, die bei einer historischen Analyse auf der Basis von Polizeiarchiven zur Vorsicht in der Auslegung ermahnt. ${ }^{69}$ In Bezug auf Tabergers eigene Perspektive auf sein „Schaufensterkriegstheater" lassen sich nur sehr vage komplementäre Vermutungen anstellen. Die Polizeikommissare erwogen beispielsweise gar nicht, daß Taberger vielleicht lediglich, wie seine anderen Hannoveraner Mitbürger, von Entsetzen und Trauer um die verletzten und verschollenen Soldaten ergriffen war und deshalb die Sarginszenierung vorgenommen

\footnotetext{
${ }^{63}$ Schreiben von Bongars an die Polizeikommissare Frömbling und Grahn $\mathrm{N}^{\circ}$ 492, 28. Febr. 1813, RNB, F 993 Arch. Westf., $\mathrm{N}^{\circ} 13850$.

64 Vgl. ebd.

${ }^{65}$ Schreiben von Bongars an Frantz, den Präfekten des Aller-Departements, $\mathrm{N}^{\circ}$ 493, 28. Febr. 1813, ebd.

66 Schreiben von Bongars an die Polizeikommissare Grahn und Frömbling N 565, 6. März 1813, RNB, F 993 Arch. Westf., $\mathrm{N}^{\circ} 13850$.

67 Vgl. ebd.

68 Schreiben von Bongars an die Polizeikommissare Grahn und Frömbling N 665, 16. März 1813, ebd.

69 Vgl. Algazi, Kulturkult, S. 111, 117; Bachmann-Medick, Kultur als Text, S. $33 \mathrm{ff}$.
} 
hatte. Möglicherweise schloß er sich mit seiner Handlung den bewegten Solidaritätsbekundungen seiner Mitbürger an. ${ }^{70}$ So könnte seine Handlung eher als Andenken an die Toten der großen Armee denn als antifranzösische Kritik zu deuten sein. Die Polizeikommissare waren aber nicht bereit, in Tabergers Handlung ein zusätzliches Zeichen der Menschlichkeit eines Hannoveraner Bürgers zu erblicken, der den nicht zurückkehrenden Soldaten mit seinem Sarg ein Denk- und Mahnmal gesetzt haben könnte. In ihrem Lob über das Verhalten der Hannoveraner schlossen die Polizeikommissare offensichtlich Taberger aus. Zum Schluß ihres Berichtes vom 16. Februar 1813 bekannten sie:

„Jamais un rapport nous a fait une plus grande satisfaction, que celui-ci ... qui prouve qu'on doit juger les Hanovriens d'après leurs faits et pas seulement d'après leurs mots ou leurs discours politiques".71

Sie zeigten sich beeindruckt von der Wohltätigkeit der Hannoveraner, sprachen jedoch wenige Tage darauf Taberger von vornherein die gelobte Empathie seiner Mitbürger $a_{b .}{ }^{72}$ Die Handlung Tabergers könnte - und diese Vermutung lag den Polizeikommissaren sicherlich näher - auch aus der Erwartung und Hoffnung auf den Tod Napoleons gewachsen sein. Einige Hannoveraner werden sicherlich ebenfalls geneigt gewesen sein, in seiner Sarginszenierung die Hoffnung auf den Tod Napoleons zu erkennen, die ihnen als Abhilfe der Not der Zeit erschien.

Die Mehrdeutigkeit von Tabergers Handlung und die Unbestimmbarkeit seiner Intention machen gerade die Analyse seiner Affäre mit der Polizei interessant in Hinsicht auf die Möglichkeiten, die außersprachliche Kommunikationsformen für die westphälischen Bürger boten. Das Bild eines unschuldigen und naiven oder eines hinterlistigen und zeitkritischen Tabergers läßt sich nicht bestimmen. Für die Analyse der Affäre Taberger bringt es wenig, wenn eine Festlegung im nachhinein erzielt wird. Die Beurteilung seiner Tat durch die Polizeikommissare bzw. der Kontext, in dem diese Urteilsfindung für ihn nachteilig ausfiel, macht die Vielfalt der Deutungsmöglichkeiten, die sich den Zeitgenossen boten, deutlich.

Was läßt nun die undeutliche Interpretation durch die Polizeikommissare in den Verhören mit Taberger an Vermutungen zu über die Wahrnehmung der Sarginszenierung durch die übrigen Hannoveraner? Die Befürchtungen der Polizeikommissare gingen dahin, daß jeder vorbeiziehende Passant sich der Sarginszenierung annehmen könne. Jeder Zuschauer war in der Lage, die Sarginszenierung in seinem Sinn zu deuten. Das Militär konnte daran womöglich Anstoß nehmen. Die Schaufensterinszenierung mochten sie beispielsweise als Andenken an die geschlagene Armee Napo-

70 Vgl. Schreiben von Bongars an die Polizeikommissare Grahn und Frömbling $\mathrm{N}^{\circ}$ 438, 22. Febr. 1813, und Schreiben $\mathrm{N}^{\circ} 458$, RNB, F 993 Arch. Westf., K. 13, $\mathrm{N}^{\circ}$ 7604-7687, hier $\mathrm{N}^{\circ}$ 7631-7632 von Bongars an die Polizeikommissare Grahn und Frömbling, 24. Febr. 1813, RNB, F 993 Arch. Westf., N 13850.

71 Schreiben der Polizeikommissare Grahn und Frömbling an Bongars, 16. Febr. 1813, RNB, F 993 Arch. Westf., K. 13, $\mathrm{N}^{\circ} 7604-7687$, hier $\mathrm{N}^{\circ} 7631$.

72 Das Beispiel der wohltätigen Hannoveraner gegenüber der napoleonischen Armee zeigt übrigens, daß das in der Historiographie der Befreiungskriege viel beschworene nationale Ressentiment der Deutschen gegenüber den Franzosen hier keine Rolle in ihrem Verhalten spielen konnte. Vgl. dazu Baldet, S. 143, 164. 
leons verstehen. Entscheidend ist, daß eine Vielfalt von Umwandlungs- und Aneignungsprozessen von den Polizeikommissaren vorausgesetzt wurde. Tabergers Handlung bot jedenfalls genug Zweideutigkeit, um jedem Passanten seine eigene Interpretation der Aufstellung zu ermöglichen.

\section{Schlußbetrachtungen}

Die Affäre des Zinngießers Taberger ist interessant, weil vielfache Deutungsprozesse in den Quellen nachvollziehbar werden. Im ersten Bericht des Polizeikommissars Frömbling steht, daß er erst durch eine Anzeige von der Aufstellung im Schaufenster von Taberger erfahren habe. Seiner Interpretation der Aufstellung als Parodie des Rußlandzugs war der Deutungsprozeß eines Denunzianten vorausgegangen. Befürchtet wurde, daß manche - das Militär - die Sarginszenierung als anstößig deuten würden, andere - die Hannoveraner - den Sarg möglicherweise als Todesnachricht, als Kritik am napoleonischen Feldzug oder als Prophezeiung des Untergangs der napoleonischen Herrschaft sehen würden.

Die Hinzuziehung von kontextualisierenden Quellenauszügen ermöglichte unter anderem, auf die hohe Konjunktur für Gerüchte vom Tod Napoleons und der Koexistenz von Todes- und Lebensnachrichten in Kriegszeiten aufmerksam zu machen. Mit diesem wechselhaften und unberechenbaren Hintergrund werden die mehrfachen Deutungsmöglichkeiten, die die Inszenierung Tabergers für die Hannoveraner und ihre Polizeikommissare beinhaltete, nachvollziehbar. Die interpretatorische Arbeit der Polizeikommissare implizierte, daß Tabergers Sarginszenierung den Zeitgeist zitierte.

Der Fall Taberger war zwar aus der Perspektive der Polizeimitglieder eindeutig. Allerdings ergibt sich aus den vielen Verhören, die mit Taberger geführt wurden, eine gravierende Verschiebung zwischen seinen Handlungen und seinen Worten - seine falsche Aussage bezüglich Delarochette trägt dazu bei. Sie differierten derart, daß seine Intentionen unklar bleiben. Dieses Problem in der Quellenauslegung, die aus der Diskrepanz zwischen Worten, Handlungen und Intentionen entsteht, ist insbesondere in Fällen der außersprachlichen Kommunikation bemerkenswert. In dieser Unbestimmbarkeit bestand wahrscheinlich die Dynamik außersprachlicher Kommunikation für die Zeitgenossen. Die westphälischen Bürger wurden bei Phänomenen außersprachlicher Kommunikation vor gravierende Deutungsprobleme gestellt. Diejenigen unter ihnen, die nicht mit den Polizeimitgliedern in Konflikt über die Deutungsmöglichkeiten ihrer Handlungen zu geraten wünschten, werden allerdings aus der Geschichte Tabergers gelernt haben. Seine Geschichte erzielte eine gewisse Öffentlichkeit und machte sicherlich Schule in Hannover, sonst hätte der Polizeichef Bongars nicht so viel Fürsprache zugunsten Tabergers erhalten, als er mit ihm ein Exempel statuieren wollte. Das Handlungsrepertoire der Westphalen wird sich aus der Kenntnis über das Schicksal Tabergers entsprechend verändert haben. Prunkvolle Begräbniszeremonien waren vornehmlich bei Staatsbegräbnissen, wie demjenigen des ermordeten Kriegsministers Joseph Antoine Morio, erwünscht. ${ }^{73}$ Ein kleiner Sarg aber, in einem hannoverschen Schaufenster, war der politischen Polizei suspekt. 
Die Affäre Taberger zeigt, daß die Kommunikation im Königreich Westphalen nicht auf die Zweisprachigkeit einiger weniger Personen angewiesen war. Die „Handlung“ Tabergers bildete jenseits aller sprachlichen Barrieren eine Prokovation an die Adresse insbesondere der französischsprachigen Militärs. Die Verständigung erfolgte nicht zuletzt über Handlungen und deren Deutung. Sie benötigte somit kein sprachliches Instrumentarium. Vielmehr bedurfte es nicht einmal einer eindeutigen Aussage desjenigen, von dem die außersprachliche Botschaft ausgegangen war, um dennoch Reaktionen hervorzurufen. Der Fall Taberger beweist, daß die kommunikativen Möglichkeiten der Westphalen weit über den linguistischen „Sprechakt“ hinausgingen. Die Kommunikationspraxis erschöpfte sich neben allen Verbalisierungen nicht in Mimik und Gestik, sondern ließ sich z.B. in anspielungsreichem Arrangement von Alltagsgegenständen mal nach tradierten Mustern, mal erfinderisch gestalten.

73 Vgl. Das Begräbnis des Grafen Morio, 1811, GStAPK, V, HA., Rep. VI., II. A., N 58; vgl. Schreiben N $^{\circ}$ 5467 des Innenministers von Wolffradt an Mercier, Generalpolizeikommissar in Kassel, GStAPK, V, HA., Rep. VI., II. A., NR 7, B1. 139. 